\title{
Was tun? Sicherheitslücken in Hardware
}

Für den Umgang mit Software-Schwachstellen gibt es heute Routinen: die werden entdeckt, veröffentlicht und schnellst möglichst durch ein Update des Software-Entwicklers oder -Anbieters beseitigt. Dazu gibt es zahlreiche internationale, regionale oder nationale Vorschriften. Im weiteren Sinne gehören dazu die Meldepflichten für IT-Sicherheitsvorfälle gemäß dem deutschen IT-Sicherheitsgesetz oder der europäischen NIS-Richtlinie ${ }^{1}$.

Wesentlich komplexer ist die Situation bei dem Versuch mit Hardware-Schwachstellen plausibel und transparent umzugehen.

Das Thema begleitet die IT-Welt seit Langem. Im Grunde geht es immer um die Frage, ob zugesicherte Sicherheitseigenschaften erfüllt werden. Das ist zunächst immer eine Momentaufnahme, die vom Stand des Wissen und des Nachweises oder der Abwehr von Angriffen bestimmt wird. Paul Kocher hat 1996 mit der Publikation Timing Attacks on Implementations of Diffie-Hellman, RSA, DSS, and Other Systems einen Stein ins Wasser geworfen und bis dahin unbeachtete Angriffe über Seitenkanäle sichtbar gemacht. In den Folgejahren war zunächst nur das akademische Interesse an der Möglichkeit, auf diese Weise Informationen über private kryptografische Schlüssel zu erlangen, geweckt. Mit der zunehmenden Perfektionierung der Angriffe gewannen diese mehr und mehr praktische Bedeutung. Das Interesse an Seitenkanalattacken hat sich inzwischen zu einem nachhaltigen Forschungsgebiet entwickelt, das für die praktische IT-Sicherheit relevant ist. Es ist ein regelrechter Wettlauf entstanden, die ständige Entwicklung von Angriffen und Gegenmaßnahmen schlägt sich in aufwändigen Sicherheitszertifizierungen nieder.

Inzwischen sind nicht nur noch kryptographische Schlüssel von Seitenkanälen betroffen. Die Annahme, dass die Schutzmechanismen von PC-Prozessoren verhindern, dass Prozesse Daten anderer Prozesse ausspähen, ist defacto nicht mehr haltbar. Denn Computer und Prozessoren verraten selbst über mehr oder weniger schwer erkennbare Kanäle Information über die Operationen, die sie bearbeiten. „Spectre“ und „Meltdown“ haben diese Tatsache 2018 deutlich gemacht.

1 DIRECTIVE (EU) 2016/1148 OF THE EUROPEAN PARLIAMENT AND OF THE COUNCILof 6 July 2016 concerning measures for a high common level of security of network and information systems across the Union
Beeindruckend ist das bisher beschreibbare Spektrum von Methoden aussichtsreicher Seitenkanalattacken. Neben der Laufzeit können beispielsweise Stromverbrauch, elektromagnetische, Wärme- bzw. Photonen-Abstrahlung gemessen und ausgewertet werden. Besonders aufschlussreich ist auch das gezielte Auslösen von Fehlern bei der Verarbeitung von sensitiven Daten, z.B durch das von Software provozierte, zufällige, fehlerhafte Kippen von Bits im Speicher.

Es sind also physikalisch und objektiv nicht vermeidbare Effekte, die sich mit im Allgemeinen hohen technischen und datenanalytischen Aufwendungen als Forschungsgebiete anbieten. Prinzipbedingt sind Seitenkanäle nicht vermeidbar: Wo gehobelt wird, fallen Späne. Solange für die Verarbeitung der Daten Elektronen bewegt werden, sind damit verbundene physikalische Effekte mess- und auswertbar. Entsprechend herausfordernd ist es für die Hersteller, die Hardware so zu gestalten, dass die messbaren Beobachtungen nicht genügend Informationen liefern, um die verarbeiteten Daten zurückzugewinnen. Das Prinzip Security-by-Obscurity ist noch an der Tagesordnung und bleibt auch künftig unverzichtbar.

Tatsache ist: Sowohl der Endnutzer von IT-Komponenten und Anwendungen als auch die Betreiber von IT-Systemen und -Infrastrukturen sind bisher nicht in der Lage gegen Hardware-Sicherheitslücken erfolgversprechend vorzugehen. Deshalb ist der Ratschlag die aktuell benutze Hardware gegen eine möglicherweise resistente Generation auszutauschen aufwändig, aber plausibel. Doch Hardware wird sich weiterentwickeln. Nicht nur, dass Zertifizierung inzwischen ein Umfang erreicht hat, der die üblichen Produktzyklen sprengt. Neue Angriffe und bessere Messund Analysemethoden machen auch vor zertifizierter Hardware keinen Halt. Ein aufwändig zertifiziertes Produkt gleich wieder gegen die nächste Generation auszutauschen wird auf lange Sicht nicht funktionieren. Hier werden intelligente Updatekonzepte benötigt, um auf neue Bedrohungen angemessen zu reagieren.

Die dargestellten komplexen Zusammenhänge machen auch verständlich, weshalb die juristische Aufbereitung der Konsequenzen von Hardware-Sicherheitslücken noch in den Anfängen steckt. 